\title{
Superluminal X-shaped beams propagating without distortion along a coaxial guide ${ }^{(\dagger)}$
}

\author{
Michel Zamboni-Rached, K. Z. Nóbrega \\ DMO-FEEC, State University at Campinas, Campinas, S.P., Brasil. \\ Erasmo Recami \\ Facoltà di Ingegneria, Università statale di Bergamo, Dalmine (BG), Italy; \\ INFN-Sezione di Milano, Milan, Italy; and \\ C.C.S., State University at Campinas, Campinas, S.P., Brasil. \\ and \\ Hugo Enrique Hernández-Figueroa \\ DMO-FEEC, State University at Campinas, Campinas, S.P., Brasil.
}

\begin{abstract}
In a previous paper we showed that localized Superluminal solutions to the Maxwell equations exist, which propagate down (non-evanescence) regions of a metallic cylindrical waveguide. In this paper we construct analogous non-dispersive waves propagating along coaxial cables. Such new solutions, in general, consist in trains of (undistorted) Superluminal "X-shaped" pulses. Particular attention is paid to the construction of finite total energy solutions. Any results of this kind may find application in the other fields in which an essential role is played by a wave-equation (like acoustics, geophysics, etc.).

PACS nos.: 03.50.De ; 41.20;Jb ; 83.50.Vr ; 62.30.+d； 43.60.+d； 91.30.Fn ; 04.30.Nk； 42.25.Bs； 46.40.Cd； 52.35.Lv .
\end{abstract}

Keywords: Wave equations; Wave propagation; Localized beams; Superluminal waves; Coaxial cables; Bidirectional decomposition; Bessel beams; X-shaped waves; Maxwell equations; Microwaves; Optics; Special relativity; Coaxial metallic waveguides; Acoustics; Seismology; Mechanical waves; Elastic waves; Guided gravitational waves

(†) Work partially supported by FAPESP (Brasil) and by INFN, MIUR (Italy). E-mail addresses for contacts: recami@mi.infn.it [ER]; giz.r@uol.com.br [MZR]. 


\section{1. - Introduction}

In a previous paper[1] we constructed localized Superluminal solutions to the Maxwell equations propagating along (non-evanescent regions of) a metallic cylindrical waveguide. In the present paper we are going to show that analogous solutions exist even for metallic coaxial cables. Their interest is due to the fact that they propagate without distortion with Superluminal group-velocity.

Let us recall that already in 1915 Bateman[2] showed Maxwell equations to admit (besides of the ordinary solutions, endowed with speed $c$ in vacuum) of wavelet-type solutions, endowed in vacuum with group-velocities $0 \leq v \leq c$. But Bateman's work went practically unnoticed, with the exception of a few authors as Barut et al.[3]. (Incidentally, Barut et al. even constructed a wavelet-type solution[4] traveling with Superluminal group-velocity $V>c$ ).

In recent times, however, many authors started to discuss the circumstance that all wave equations admit of solutions with $0 \leq v \leq \infty$ : see, e.g., refs.[5]. Most of those authors confined themselves to investigate (sub- or Super-luminal) non-dispersive solutions propagating in the open space only: namely, those solutions that had been called "undistorted progressive waves" by Courant \& Hilbert[6].

Among localized solutions, the most interesting appeared to be the "X-shaped waves", which, predicted long ago to exist within Special Relativity in its extended version[7,8], had been mathematically constructed by Lu et al.[9] for acoustic waves, and by Ziolkowski et al.[10] and by Recami[11] for electromagnetic waves.

Let us stress that such "X-shaped" localized solutions are Superluminal (i.e., travel with a speed larger than $c$ in vacuum) in the electromagnetic case; and are "Super-sonic" (i.e., travel with a speed larger than the sound-speed in the medium) in the acoustic case. The first authors to produce experimentally X-shaped waves were Lu et al.[13] for acoustics, Saari et al.[14] for optics, and Mugnai et al.[15] for microwaves. Let us also emphasize, incidentally, that all such solutions can have an interesting role even in seismology, and probably in the gravitational wave sector.

Notwithstanding all that work[16], it is not well understood yet what solutions let us now confine ourselves, for simplicity, to Maxwell equations and to electromagnetic waves only - have to enter into the play in realistic experiments using waveguides, optical 
fibers, etc.

\section{2. - The case of a cylindrical waveguide}

As we already mentioned, in ref.[1] we constructed, for the TM (transverse magnetic) case, localized solutions to the Maxwell equations which propagate (undistorted) with Superluminal speed along a cylindrical waveguide. Let us take advantage of the present opportunity for calling further attention to two points, which received just a mention in ref.[1], with regard to eq.(9) and Fig.2 therein. Namely, let us here stress that:

(i) those solutions consist in a train of pulses like the one depicted in Fig.2 of ref.[1]; and that

(ii) each of such pulses is X-shaped.

A more complete representation of the TM (and TE) non-dispersive waves, traveling down a cylindrical waveguide, will be forwarded elsewhere.

\section{3. - The case of a coaxial cable}

Let us now examine the case of a coaxial cable (a metallic coaxial waveguide, to fix our ideas), that is, of the region delimited by two cylinders with radius $\rho=r_{1}$ and $\rho=r_{2}$, respectively, and axially symmetric with respect to the $z$-axis: see Fig.1. We shall consider in this article both the TM case, characterized by the Dirichlet boundary conditions[17] (for any time instant $t$ )

$$
E_{z}\left(\rho=r_{1} ; t\right)=0 ; \quad E_{z}\left(\rho=r_{2} ; t\right)=0 ;
$$

and the TE (transverse electric) case, characterized by the Neumann boundary conditions[17] (for any $t$ )

$$
\frac{\partial}{\partial \rho} H_{z}\left(\rho=r_{1} ; t\right)=0 ; \quad \frac{\partial}{\partial \rho} H_{z}\left(\rho=r_{2} ; t\right)=0
$$


To such aims, we shall first generalize a theorem due to Lu et al.[18] (stated and demonstrated below, in the Appendix), which showed how to start from a solution holding in the plane $(x, y)$ for constructing a three-dimensional solution rigidly moving along the $z$-axis with Superluminal speed $V$. The Lu et al.'s theorem was valid for the vacuum. In ref.[1] we set forth its generalization for a cylindrical waveguide, while here we are going to extend it, as we said above, for a coaxial cable. Let us first recall what Lu et al.'s theorem is about. If we assume that $\psi(\boldsymbol{\rho} ; t)$, with $\boldsymbol{\rho} \equiv(x, y)$, is a solution of the two-dimensional homogeneous wave equation

$$
\left(\partial_{x}^{2}+\partial_{y}^{2}-\frac{1}{c^{2}} \partial_{t}^{2}\right) \psi(\boldsymbol{\rho} ; t)=0
$$

then, by applying the transformations

$$
\boldsymbol{\rho} \longrightarrow \boldsymbol{\rho} \sin \theta ; \quad \text { and } \quad t \longrightarrow t-z\left(\frac{\cos \theta}{c}\right)
$$

the angle $\theta$ being fixed, with $0<\theta<\pi / 2$, one gets[18] that $\psi(\boldsymbol{\rho} \sin \theta ; t-z \cos \theta / c)$ is now a solution of the three-dimensional homogeneous wave-equation

$$
\left(\boldsymbol{\nabla}^{2}-\frac{1}{c^{2}} \partial_{t}^{2}\right) \psi\left(\boldsymbol{\rho} \sin \theta ; t-z \frac{\cos \theta}{c}\right)=0
$$

where now $\boldsymbol{\nabla}^{2} \equiv \partial_{x}^{2}+\partial_{y}^{2}+\partial_{z}^{2} ; \boldsymbol{\rho} \equiv(x, y)$.

The mentioned theorem holds for the free case, so that in general it does not hold when introducing boundary conditions. We shall see, however, that it can be extended even to the case of a two-dimensional solution $\psi$ valid on an annular domain, $a \leq \rho \leq b ; \rho \equiv|\boldsymbol{\rho}|$, with either the (Dirichlet) boundary conditions

$$
\psi(\rho=a ; t)=\psi(\rho=b ; t)=0,
$$

or the (Neumann) boundary conditions

$$
\frac{\partial}{\partial \rho} \psi(\rho=a ; t)=\frac{\partial}{\partial \rho} \psi(\rho=b ; t)=0 .
$$


Let us notice right now that transformations (4), with condition (1') or (2'), lead to a (three-dimensional) solution rigidly traveling with Superluminal speed $V=c / \cos \theta$ inside a coaxial cable with internal and external radius equal (no longer to $a, b$, but) to $r_{1}=a / \sin \theta>a$ and $r_{2}=b / \sin \theta>b$, respectively. The same procedure can be applied also in other cases, provided that the boundary conditions depend on $x, y$ only: as in the case, e.g., of a cable with many cylindrical (empty) tunnels inside it.

\section{4. - The transverse magnetic (TM) case}

Let us go back to the two-dimensional equation (3) with the boundary conditions $\left(1^{\prime}\right)$. Let us choose for instance the simple initial conditions $\psi(\rho ; t=0) \equiv \phi(\rho)$ and $\partial \psi / \partial t \equiv \xi(\rho)$ at $t=0$, where

$$
\phi(\rho)=\delta\left(\rho-\rho_{0}\right) ;\left.\quad \xi(\rho)\right|_{t=0}=0
$$

with

$$
a<\rho_{0}<b .
$$

Following a method similar to the one in ref.[1], and using the boundary conditions $\left(1^{\prime}\right)$, in cylindrical co-ordinates and for axial symmetry one gets solutions to eq.(3) of the type $\psi=\sum R_{n}(\rho) T_{n}(t)$ in the following form:

$$
2 \psi(\rho ; t)=\sum_{n=1}^{\infty} R_{n}(\rho)\left[A_{n} \cos \omega_{n} t-B_{n} \sin \omega_{n} t\right]
$$

where the functions $R(\rho)$ are

$$
R_{n}(\rho) \equiv N_{0}\left(k_{n} a\right) J_{0}\left(k_{n} \rho\right)-J_{0}\left(k_{n} a\right) N_{0}\left(k_{n} \rho\right)
$$

quantities $N_{0}$ and $J_{0}$ being the zeroth-order Neumann and Bessel functions, respectively; and where the characteristic angular frequencies[19] can be evaluated numerically, they 
being solutions to the equation $\left[\omega_{n}=c k_{n}\right]$

$$
\frac{J_{0}\left(k_{n} a\right)}{N_{0}\left(k_{n} a\right)}=\frac{J_{0}\left(k_{n} b\right)}{N_{0}\left(k_{n} b\right)}
$$

The initial conditions (6) imply that $\sum A_{n} R_{n}(\rho)=\delta\left(\rho-\rho_{0}\right)$, and $\sum B_{n} R_{n}(\rho)=0$, so that all the coefficients $B_{n}$ vanish, and eventually one obtains the two-dimensional solution

$$
\Psi_{2 \mathrm{D}}(\rho ; t)=\sum_{n=1}^{\infty} A_{n} R_{n}(\rho) \cos \omega_{n} t
$$

with

$$
\begin{aligned}
2 A_{n}= & \left\{-a^{2}\left[N_{0}\left(k_{n} a\right) J_{1}\left(k_{n} a\right)-J_{0}\left(k_{n} a\right) N_{1}\left(k_{n} a\right)\right]^{2}+\right. \\
& \left.+b^{2}\left[N_{0}\left(k_{n} a\right) J_{1}\left(k_{n} b\right)-J_{0}\left(k_{n} a\right) N_{1}\left(k_{n} b\right)\right]^{2}\right\}^{-1} R_{n}\left(\rho_{0}\right)
\end{aligned}
$$

One can notice that the present procedure is mathematically analogous to the analysis of the free vibrations of a ring-shaped elastic membrane[19].

For any practical purpose, one has of course to take a finite number $N$ of terms in expansion (10). In Fig.2 we show, e.g., the two-dimensional functions $\left|\Psi_{2 \mathrm{D}}(\rho ; t)\right|^{2}$ of eq.(10) for fixed time $(t=0)$ and for $N=10$ (dotted line) or $N=40$ (solid line). Notice that when the value $N$ is finite, the first one of conditions (6) is no longer a delta function, but represents a physical wave, which nevertheless is still clearly bumped (Fig.2). It is rather interesting that, for each value of $N$, one meets a different (physical) situation; at the extent that we obtain infinite many different families of three-dimensional solutions, by varying the truncating value $N$ in eq.(12) below.

Actually, by the transformations (4) we arrive from eq.(10) at the three-dimensional Superluminal non-dispersive solution $\Psi_{3 \mathrm{D}}$, propagating without distortion along a metallic coaxial waveguide, i.e., down a coaxial cable $[V>c]$ :

$$
\Psi_{3 \mathrm{D}}(\rho ; z-V t)=\sum_{n=1}^{\infty} A_{n} R_{n}(\rho \sin \theta) \cos \left[k_{n}(z-V t) \cos \theta\right]
$$

which is a sum over different propagating modes. The fact that $V=c / \cos \theta>c$ means 
(once more) that the group-velocity of our pulses is Superluminal. For simplicity, in our Figures we shall put $z-V t \equiv \eta$.

Let us notice that transformations (4), which change - as we already know - $a$ into $r_{1}=a / \sin \theta$ and $b$ into $r_{2}=b / \sin \theta$, are such that the maximum of $\Psi_{3 \mathrm{D}}$ is got for the value $\rho_{0} / \sin \theta$ of $\rho$. However, solution (12) does automatically satisfy on the cylinders with radius $r_{1}$ and $r_{2}$ the conditions $\left[\Psi_{3 \mathrm{D}} \equiv E_{z}\right]$ :

$$
\Psi_{3 \mathrm{D}}(\rho=a / \sin \theta, z ; t)=\Psi_{3 \mathrm{D}}(\rho=b / \sin \theta, z ; t)=0 .
$$

Till now, $\Psi_{3 \mathrm{D}}$ has represented the electric field component $E_{z}$. Let us add that in the TM case[20]:

$$
\boldsymbol{E}_{\perp}=i \frac{c V}{V^{2}-c^{2}} \sum_{n=1}^{\infty} \frac{1}{k_{n}} \nabla_{\perp} \Psi_{3 \mathrm{D}}
$$

where

$$
\frac{c V}{V^{2}-c^{2}} \equiv \frac{\cos \theta}{\sin ^{2} \theta}, \quad k_{n}=\omega_{n} / c
$$

and

$$
\boldsymbol{H}_{\perp}=\varepsilon_{0} \frac{V}{c} \hat{\boldsymbol{z}} \wedge \boldsymbol{E}_{\perp}
$$

As we mentioned above, for any truncating value $N$ in expansion (10), we get a different physical situation: In a sense, we excite in a different way the two-dimensional annular membrane, obtaining (via Lu et al.'s theorem) different three-dimensional solutions, which correspond[1] to nothing but summation (12) truncated at the value $N$.

In Figs.3a,b, we show a single (X-shaped) three-dimensional pulse $\Psi_{3 \mathrm{D}}$ with $\theta=84^{\circ}$, and $N=10$ or $N=40$, respectively.

In Fig.4, by contrast, we depict a couple of elements of the train of X-shaped pulses represented by eq.(12), for $\theta=45^{\circ}$ and $N=40$.

* Let us recall that the group-velocity is well defined only when the pulse has a clear bump in space; but it can be calculated by the approximate relation $v_{g} \simeq \mathrm{d} \omega / \mathrm{d} \beta$, quantity $\beta$ being the wavenumber, only when some extra conditions are satisfied (namely, when $\omega$ as a function of $\beta$ is also clearly bumped). In the present case the group-velocity is very well defined, but cannot be evaluated through that simple relation, since $\omega$ is a discrete function of $\beta$ : cf. eq.(9) and Sect.6, eq.(22), below. 
In Fig. 5 the orthogonal projection is moreover shown of a single pulse (of the solution in Fig.4) onto the $(\rho, z)$ plane for $t=0$, with $\theta=45^{\circ}$ and $N=40$. Quantities $\rho$ and $\eta$ are always in centimeters.

\section{5. - The transverse electric (TE) case}

In the TE case, one has to consider the two-dimensional equation (3) with the boundary conditions (2'), while the initial conditions (6) can remain the same.

As in Sect.4, one gets - still for axial symmetry in cylindrical co-ordinates - the following solution to eq.(3):

$$
2 \psi(\rho ; t)=\sum_{n=1}^{\infty} R_{n}(\rho)\left[A_{n} \cos \omega_{n} t-B_{n} \sin \omega_{n} t\right]
$$

where now the functions $R_{n}(\rho)$ are

$$
R_{n}(\rho) \equiv N_{1}\left(k_{n} a\right) J_{0}\left(k_{n} \rho\right)-J_{1}\left(k_{n} a\right) N_{0}\left(k_{n} \rho\right)
$$

defined in terms of different values of $k_{n}$. In fact, the characteristic (angular) frequencies are now to be obtained by the new relation

$$
\frac{J_{1}\left(k_{n} a\right)}{N_{1}\left(k_{n} a\right)}=\frac{J_{1}\left(k_{n} b\right)}{N_{1}\left(k_{n} b\right)} .
$$

Again, the initial conditions (6) entail that $\sum A_{n} R_{n}(\rho)=\delta\left(\rho-\rho_{0}\right)$, and $\sum B_{n} R_{n}(\rho)=$ 0 , so that all the coefficients $B_{n}$ vanish, and one gets the two-dimensional solution

$$
\Psi_{2 \mathrm{D}}(\rho ; t)=\sum_{n=1}^{\infty} A_{n} R_{n}(\rho) \cos \omega_{n} t
$$

where the coefficients $A_{n}$ are given by

$$
\begin{aligned}
2 A_{n}= & \left\{-a^{2}\left[N_{1}\left(k_{n} a\right) J_{0}\left(k_{n} a\right)-J_{1}\left(k_{n} a\right) N_{0}\left(k_{n} a\right)\right]^{2}+\right. \\
& \left.+b^{2}\left[N_{1}\left(k_{n} a\right) J_{0}\left(k_{n} b\right)-J_{1}\left(k_{n} a\right) N_{0}\left(k_{n} b\right)\right]^{2}\right\}^{-1} R_{n}\left(\rho_{0}\right) .
\end{aligned}
$$


In this case one obtains, by transformations (4), the Superluminal non-dispersive three-dimensional solution

$$
\Psi_{3 \mathrm{D}}(\rho ; z-V t)=\sum_{n=1}^{\infty} A_{n} R_{n}(\rho \sin \theta) \cos \left[k_{n}(z-V t) \cos \theta\right]
$$

propagating along the metallic coaxial waveguide with group-velocity $V=c / \cos \theta>c$. The present solution (16) satisfies the boundary conditions

$$
\left.\frac{\partial}{\partial \rho} \Psi_{3 \mathrm{D}}(\rho, z, t)\right|_{\rho=\frac{a}{\sin \theta}}=\left.\frac{\partial}{\partial \rho} \Psi_{3 \mathrm{D}}(\rho, z, t)\right|_{\rho=\frac{b}{\sin \theta}}=0
$$

where now $\Psi_{3 \mathrm{D}} \equiv H_{z}$. The transverse components, in the TE case, are given[20] by

$$
\boldsymbol{H}_{\perp}=\frac{-c V}{V^{2}-c^{2}} \sum_{n=1}^{\infty} \frac{1}{k_{n}} \sin \left[k_{n}(z-V t) \cos \theta\right] \boldsymbol{\nabla}_{\perp} R_{n}(\rho \sin \theta)
$$

and

$$
\boldsymbol{E}_{\perp}=-\mu_{0} \frac{V}{c} \hat{\boldsymbol{z}} \wedge \boldsymbol{H}_{\perp}
$$

In Fig.6 we plot our function $\Psi_{2 \mathrm{D}}$ with $N=10$ (dotted line) or $N=40$ (solid line). In Fig.7 there are depicted, by contrast, two elements of the train of X-shaped pulses represented by eq.(16), with $\theta=60^{\circ}$, for $N=40$ only. In Fig.8, at last, we show the orthogonal projection (of a single pulse of the solution in Fig.7) onto the plane $(\rho, z)$ for $t=0$, with $\theta=60^{\circ}$ and $N=40$. Quantities $\rho$ and $\eta$ are in $\mathrm{cm}$.

\section{6. - Rederivation of our results from the standard theory of waveguide prop- agation}

Lu's theorem is certainly a very useful tool to build up localized solutions to Maxwell equations: nevertheless, due to the novelty of our previous results, it may be worthwhile to outline an alternative derivation[1] of them which can sound more familiar. To such an aim, we shall follow the procedure introduced in ref.[1]. 
For the sake of simplicity, let us limit ourselves to the domain of TM (transverse magnetic) modes. When a solution in terms of the longitudinal electric component, $E_{z}$, is sought, one has to deal with the boundary condition $E_{z}=0$; we shall look, moreover, for axially symmetric solutions (i.e., independent of the azimuth variable, $\varphi$ ): Such choices could be easily generalized, just at the cost of increasing the mathematical complexity. Quantity $E_{z}$ is then completely equivalent to the scalar variable $\Psi \equiv \Psi_{3 \mathrm{D}}$ used in the previous analysis.

Let us look for solutions of the form[1]

$$
E_{z}(\rho, z ; t)=K R(\rho) \exp \left[i\left(\frac{\omega z \cos \theta}{c}-\omega t\right)\right]
$$

where $R(\rho)$ is assumed to be a function of the radial coordinate $\rho$ only, and $K$ is a normalization constant. Here we call $c$ the velocity of light in the medium filling the coaxial waveguide, supposing it nondispersive. The (angular) frequency $\omega$ is for the moment arbitrary.

By inserting expression (18) into the Maxwell equation for $E_{z}$, one obtains[1]

$$
\rho^{2} \frac{\mathrm{d}^{2} R(\rho)}{\mathrm{d} \rho^{2}}+\rho \frac{\mathrm{d} R(\rho)}{\mathrm{d} \rho}+\rho^{2} \Omega^{2} R(\rho)=0 ; \quad \Omega \equiv \frac{\omega \sin \theta}{c}
$$

whose only solution, which is finite on the waveguide axis, is $R(\rho)=$ $N_{0}(\omega a / c) J_{0}(\omega \rho \sin \theta / c)-J_{0}(\omega a / c) N_{0}(\omega \rho \sin \theta / c)$, which is analogous to eq. (8).

By imposing the boundary conditions $R(\rho)=0$ for $\rho=r_{1}=a / \sin \theta$ and $\rho=r_{2}=$ $b / \sin \theta$, one gets the acceptable frequencies from the characteristic equation:

$$
\frac{J_{0}\left(\omega_{n} a / c\right)}{N_{0}\left(\omega_{n} a / c\right)}=\frac{J_{0}\left(\omega_{n} b / c\right)}{N_{0}\left(\omega_{n} b / c\right)}
$$

so that one has a different function $R_{n}(\rho)$ for each value of $\omega_{n}$. Therefore, assuming[1] an arbitrary parameter $\theta$, we find that, for every mode supported by the waveguide and labeled by the index $n$, there is just one frequency at which the assumed dependence (18) on $z$ and $t$ is physically realizable. Let us show such a solution to be the standard one known from classical electrodynamics. In fact, by inserting[1] the allowed frequencies $\omega_{n}$ into the complete expression of the mode, we have: 


$$
E_{z}^{n}(\rho, z ; t)=K R_{n}(\rho) \exp \left[i\left(\frac{\omega_{n} z \cos \theta}{c}-\omega_{n} t\right)\right]
$$

But the generic solution for (axially symmetric) $\mathrm{TM}_{0 n}$ modes[21] in a coaxial metallic waveguide is $\left[\Omega_{n} \equiv \omega_{n} \sin \theta / c\right]$ :

$$
E_{z}^{\mathrm{TM}_{0 n}}=K R_{n}(\rho) \exp \left[i\left(\beta\left(\omega_{n}\right) z-\omega_{n} t\right)\right]
$$

the wavenumber $\beta$ being a discrete function of $\omega$, with the "dispersion relations"

$$
\beta^{2}\left(\omega_{n}\right)=\frac{\omega_{n}^{2}}{c^{2}}-\Omega_{n}^{2}
$$

By identifying $\beta\left(\omega_{n}\right) \equiv \omega_{n} \cos \theta / c$, as suggested by eq.(21), and remembering the expression for $\omega_{n}$ given by eq.(20), the ordinary dispersion relation is got[1]. We have therefore verified that every term in the expansion (12) is a solution to Maxwell equations not different from the usual one.

The uncommon feature of our solution (12) is that, given a particular value of $\theta$, the phase-velocity of all its terms is always the same, it being independent of the mode index $n$ :

$$
V_{\mathrm{ph}}=\left[\frac{\beta\left(\omega_{n}\right)}{\omega_{n}}\right]^{-1}=\frac{c}{\cos \theta} .
$$

In such a case it is well-known that the group-velocity of the pulse equals the phasevelocity[22]: and in our case is the velocity tout court of the localized pulse.

With reference to Fig.9, we can easily see[1] that all the allowed values of $\omega_{n}$ can be calculated by determining the intersections of the various branches of the dispersion relation with a straight line, whose slope depends on $\theta$ only. By using suitable combinations of terms, corresponding to different indices $n$, as in our eq.(12), it is possible to describe a disturbance having a time-varying profile[1], as already shown in Figs.3-4 above. Each pulse thus displaces itself rigidly, with a velocity $v \equiv v_{\mathrm{g}}$ equal to $V_{\mathrm{ph}}$.

It should be repeated that the velocity $v$ (or group-velocity $v_{\mathrm{g}} \equiv v$ ) of the pulses corresponding to eq.(9) is not to be evaluated by the ordinary formula $v_{\mathrm{g}} \simeq \mathrm{d} \omega / \mathrm{d} \beta$ (valid for 
quasi-monochromatic signals). This is at variance with the common situation in optical and microwave communications, when the signal is usually an "envelope" superimposed to a carrier wave whose frequency is generally much higher than the signal bandwidth. In that case the standard formula for $v_{\mathrm{g}}$ yields the correct velocity to deal with (e.g., when propagation delays are studied). Our case, on the contrary, is much more reminiscent of a baseband modulated signal, as those studied in ultrasonics: the very concept of a carrier becomes meaningless here, as the discrete "harmonic" components have widely different frequencies[1].

Let us finally remark[1] that similar considerations could be extended to all the situations where a waveguide supports several modes. Tests at microwave frequencies should be rather easy to perform; by contrast, experiments in the optical domain would face the problem of the limited extension of the spectral windows corresponding to not too large attenuation, even if work[23] is in progress in many directions.

Moreover, results of the kind presented in this paper, as well as in refs.[1,11,12], may find application in the other fields in which an essential role is played by a wave-equation (like acoustics, seismology, geophysics, and relativistic quantum mechanics, possibly.).

\section{7. - How to get finite total energy solutions}

We shall go on following the standard formalism of Sect.6; what we are going to do holds, however, for both the TM and the TE case. Let us anticipate that, in order to get finite total energy solutions (FTES), we shall have to replace each characteristic frequency $\omega_{n}$ [cf. eq.(9), or eq.(14) or rather Fig.9] by a small frequency band $\Delta \omega$ centered at $\omega_{n}$, always choosing the same $\Delta \omega$ independently of $n$. In fact, since all the modes entering the Fourier-type expansion (12), or (16), possess the same phase-velocity $V_{\mathrm{ph}} \equiv V=c / \cos \theta$, each small bandwidth packet associated with $\omega_{n}$ will possess the same group-velocity $v_{\mathrm{g}}=c^{2} / V_{\mathrm{ph}}$, so that we shall have as a result a wave whose envelope travels with the subluminal group-velocity $v_{\mathrm{g}}$. However, inside the subluminal envelope, one or more pulses will be travelling with the dual (Superluminal) speed $V=c^{2} / v_{\mathrm{g}}$. Such well-localized peaks have nothing to do with the ordinary (sinusoidal) carrier-wave, and will be regarded as constituting the relevant wave. Before going on, let us mention that previous work related 
to FTESs can be found — as far as we know — only in refs.[24] and [12].

Formally, to get FTESs, let us consider the ordinary (three-dimensional) solutions for a coaxial cable:

$$
\psi_{n}(\rho, z ; t)=K_{n} R_{n}(\rho) \cos [\beta(\omega) z-\omega t]
$$

where coefficients $K_{n}$ coincide with the $A_{n}$ given by eq.(11) or eq.(15') in the TM ot TE cases, respectively; and functions $R_{n}$ are again given by eq.(8) or eq.(13'), respectively; since the values $k_{n}$,

$$
k_{n} \equiv \frac{\omega^{2}}{c^{2}}-\beta^{2}
$$

are equal to those found via the (two-dimensional) eq.(9) in the TM and via eq.(14) in the TE case, simply multiplied by $\sin \theta$ [because of the fact that, when going on from the two-dimensional membrane to the three-dimensional coaxial cable, the internal and external radia are equal (no longer to $a, b$, but) to $r_{1}=a / \sin \theta$ and $r_{2}=b / \sin \theta$ ].

Let us now consider the spectral functions

$$
W_{n} \equiv \exp \left[-q^{2}\left(\omega-\omega_{n}\right)^{2}\right]
$$

with the same weight-parameter $q$, so that $\Delta \omega$ too is the same [according to our definitions, $\Delta \omega=1 / q] ;$ and with

$$
\omega_{n} \equiv \frac{k_{n} c}{\sin \theta}
$$

quantity $\sin \theta$ having a fixed but otherwise arbitrary value. We shall construct FTESs, $\mathcal{F}(\rho, z ; t)$, of the typdit

\footnotetext{
${ }^{\dagger}$ When integrating over $\omega$ from $-\infty$ to $+\infty$ there are also the non-physical (traveling backwards in space) and the evanescent waves. But their actual contribution is totally negligible, since the weightfunctions $W_{n}$ are strongly localized in the vicinity of the $\omega_{n}$-values (which are all positive: see, e.g., Fig.9). In any case, one could integrate from 0 to $\infty$ at the price of incresing a little the mathematical complexity: we are preferring the present formalism for simplicity's sake.
} 


$$
\mathcal{F}_{3 \mathrm{D}}(\rho, z ; t)=\sum_{n=1}^{N} \int_{-\infty}^{\infty} \mathrm{d} \omega \psi_{n} W_{n},
$$

with arbitrary $N$. Notice that we are not using a single gaussian weight, but a different gaussian function for each $\omega_{n}$-value, such weights being centered around the corresponding $\omega_{n}$.

Due to the mentioned localization of the $W_{n}$ around the $\omega_{n}$-values, we can (for each value of $n$ in the above sum) expand the function $\beta(\omega)$ in the neighbourhood of the corresponding $\omega_{n}$-value:

$$
\beta(\omega) \simeq \beta_{0 n}+\left.\frac{\partial \beta}{\partial \omega}\right|_{\omega_{n}}\left(\omega-\omega_{n}\right)+\cdots
$$

where $\beta_{0 n}=\omega_{n} \cos \theta$, and the further terms are neglected since $\Delta \omega$ is assumed to be small. Notice that, because of relations (26) and (24), in eq.(28) the group-velocities, given by

$$
\frac{1}{v_{\mathrm{gn}}}=\left.\frac{\partial \beta}{\partial \omega}\right|_{\omega_{n}}
$$

are actually independent of $n$, all of them possessing therefore the same value:

$$
v_{\mathrm{g} n} \equiv v_{\mathrm{g}}=c \cos \theta
$$

By using relation (28) and the transformation of variables

$$
f_{n} \equiv \omega-\omega_{n},
$$

the integration in eq.(27) does eventually yield:

$$
\mathcal{F}_{3 \mathrm{D}}(\rho, z ; t)=\frac{\sqrt{\pi}}{q} \exp \left[-\frac{\left(z-v_{g} t\right)^{2}}{4 q^{2} v_{g}^{2}}\right] \sum_{n=1}^{\infty} A_{n} R_{n}(\rho) \cos \left[k_{n}(z-V t) \cos \theta\right],
$$

where, let us recall, $V=c^{2} / v_{\mathrm{g}}=c / \cos \theta$, and we used the identity

$$
\int_{-\infty}^{\infty} \mathrm{d} f \exp \left[-q^{2} f^{2}\right] \cos \left[f\left(v_{g}^{-1} z-t\right)\right]=\frac{\sqrt{\pi}}{q} \exp \left[-\frac{\left(v_{g}^{-1} z-t\right)^{2}}{4 q^{2}}\right] .
$$

It is rather interesting to notice that the FTES (29) is related to the X-shaped waves, since the integration in eq.(27) does eventually yield the FTES in the form: 


$$
\mathcal{F}_{3 \mathrm{D}}(\rho, z ; t)=\frac{\sqrt{\pi}}{q} \exp \left[-\frac{\left(z-v_{g} t\right)^{2}}{4 q^{2} v_{g}^{2}}\right] \mathcal{T}(\rho, z)
$$

function $\mathcal{T}(\rho, z)$ being one of our previous solutions in eq.(12) or (16) above, at our free choice.

Let us go back to the important relation (28'), and to the discussion about it started at the beginning of this Section. Let us repeat that, if we choose the $\omega_{n}$-values as in Fig.9, all our small-bandwidth packets, centered at the $\omega_{n}$ 's, get the same phase-velocity $V>c$ and therefore the same group-velocity $v_{\mathrm{g}}<c$ [since for metallic waveguides the quantities $k_{n}^{2}=\omega_{n}^{2} / c^{2}-\beta^{2}$ are constant for each mode, and $v_{\mathrm{g}} \equiv \partial \omega / \partial \beta$, so that it is $\left.V v_{\mathrm{g}}=c^{2}\right]$. This means that the envelope of solution (29)-(30) moves with slower-than-light speed; the envelope length $\Delta l$ depending on the chosen $\Delta \omega$, and being therefore proportional to $q v_{\mathrm{g}}$.

However, inside such an envelope, one gets a train of (X-shaped) pulses - having nothing to do with the ordinary carrier wave, as we already mentioned - traveling with the Superluminal speed $V$. An interesting point is that we can choose the envelope length so that it contains only one (X-shaped wave) peak: the Superluminal speed $V=c^{2} / v_{\mathrm{g}}$ of such a pulse can then be regarded as the actual velocity of the wave. In order to have just one peak inside the envelope, the envelope length is to be chosen smaller than the distance between two successive peaks of the (infinite total energy) train (12), or (16).

It should be noted, at last, that the amplitude of such a single X-shaped pulse (which remains confined inside the envelope) first increases, and afterwards decreases, while traveling; till when it practically disappears. While the considered pulse tends to vanish on the right (i.e., under the right tail of the envelope), a second pulse starts to be created on the left; and so on. From eq.(30) it is clear, in fact, that our finite-energy solution is nothing but an (infinite-energy) solution of the type in eq.(12), or in eq.(16), multiplied by a Gaussian function. In Figs.10 all such a behaviour is clearly depicted.

\section{Acknowledgements}

\footnotetext{
$\ddagger$ One may call "envelope length" the distance between the two points in which the envelope height is, for instance, $10 \%$ of its maximum height.

$\S$ Actually, they can be regarded as a sum of carrier waves.
} 
The authors acknowledge, first of all, very useful discussions with F.Fontana. For stimulating discussions, thanks are due also to V.Abate, C.Becchi, M.Brambilla, C.Cocca, R.Collina, G.C.Costa, P.Cotta-Ramusino, C.Dartora, G.Degli Antoni, A.C.G.Fernández, L.C.Kretly, J.M.Madureira, G.Pedrazzini, G.Salesi, J.W.Swart, M.T.Vasconselos, M.Villa, S.Zamboni-Rached and particularly A.Shaarawi. At last, an anonymous Referee should be thanked for useful comments.

\section{Appendix}

Let us here state, and demonstrate, the Lu's theorem, for the reader's convenience:

The theorem: $\operatorname{Be} \psi_{2 \mathrm{D}}(x, y ; t)$ a solution of the two-dimensional homogeneous wave equation

$$
\left(\partial_{x}^{2}+\partial_{y}^{2}-\frac{1}{c^{2}} \partial_{t}^{2}\right) \psi_{2 \mathrm{D}}(x, y ; t)=0
$$

On applying the transformations

$$
x \longrightarrow x^{\prime} \sin \theta ; \quad y \longrightarrow y^{\prime} \sin \theta \text { and } t \longrightarrow t^{\prime}-z^{\prime} \frac{\cos \theta}{c},
$$

the angle $\theta$ being fixed $(0<\theta<\pi / 2)$, the three-dimensional function

$$
\psi_{3 \mathrm{D}}\left(x^{\prime}, y^{\prime}, z^{\prime} ; t^{\prime}\right)=\psi_{2 \mathrm{D}}\left(x^{\prime} \sin \theta, y^{\prime} \sin \theta ; t^{\prime}-\cos \theta z^{\prime} / c\right)
$$

results to be a solution of the three-dimensional wave equation

$$
\left(\partial_{x^{\prime}}^{2}+\partial_{y^{\prime}}^{2}+\partial_{z^{\prime}}^{2}-\frac{1}{c^{2}} \partial_{t^{\prime}}^{2}\right) \psi_{3 \mathrm{D}}\left(x^{\prime}, y^{\prime}, z^{\prime} ; t^{\prime}\right)=0
$$

Its demonstration: By use of eqs.(A.2), (A.3) and of assumption (A.1), one obtains, by direct calculations, that 


$$
\begin{aligned}
& \left(\partial_{x^{\prime}}^{2}+\partial_{y^{\prime}}^{2}+\partial_{z^{\prime}}^{2}-\frac{1}{c^{2}} \partial_{t^{\prime}}^{2}\right) \psi_{3 \mathrm{D}}\left(x^{\prime}, y^{\prime}, z^{\prime} ; t^{\prime}\right)= \\
& \left(\sin ^{2} \theta \partial_{x}^{2}+\sin ^{2} \theta \partial_{y}^{2}+\frac{\cos ^{2} \theta}{c^{2}} \partial_{t}^{2}-\frac{1}{c^{2}} \partial_{t}^{2}\right) \psi_{2 \mathrm{D}}(x, y ; t)= \\
& \sin ^{2} \theta\left(\partial_{x}^{2}+\partial_{y}^{2}-\frac{1}{c^{2}} \partial_{t}^{2}\right) \psi_{2 \mathrm{D}}(x, y ; t)=0,
\end{aligned}
$$

so that the theorem gets demonstrated. 


\section{Figure Captions}

Fig.1 — Sketch of the coaxial waveguide.

Fig.2 - Square magnitude $\left|\Psi_{2 \mathrm{D}}(\rho ; t=0)\right|^{2}$ of the two-dimensional solutions in eq.(10) for fixed time $(t=0)$ and for $N=10$ (dotted line) or $N=40$ (solid line). It refers to the TM case (Dirichlet boundary conditions) with $a=1 \mathrm{~cm}, b=3 \mathrm{~cm}$ and $\rho_{0}=2 \mathrm{~cm}$ : See the text.

Figs.3 - In Figs.(a) and (b) we show the square magnitude $\left|\Psi_{3 \mathrm{D}}(\rho, \eta)\right|^{2}$ of a single (Xshaped) three-dimensional pulse of the beam in eq.(12), with $\theta=84^{\circ}, \quad r_{1}=a / \sin \theta$, $r_{2}=b / \sin \theta$ (it having been chosen $a=1 \mathrm{~cm}$ and $b=3 \mathrm{~cm}$ ), for $N=10$ and $N=40$, respectively. They refer to the TM case. Notice that $\eta \equiv z-V t$, and that the considered beam is a train of X-shaped pulses.

Fig.4 - In this figure we depict, by contrast, a couple of elements of the train of X-shaped pulses represented in the TM case by eq.(12), for $N=40$. This time the angle $\theta=45^{\circ}$ was chosen, keeping the same $a$ and $b$ values as before.

Fig.5 - The orthogonal projection is shown of a single pulse (of the solution in Fig.4, referring to the TM case) onto the $(\rho, z)$ plane for $t=0$, with $\theta=45^{\circ}$ and $N=40$.

Fig.6 - In analogy with Fig.2, the square magnitude $\left|\Psi_{2 \mathrm{D}}(\rho ; t=0)\right|^{2}$ is shown of the two-dimensional solutions in eq.(15) for fixed time $(t=0)$, and for $a=1 \mathrm{~cm}, b=3 \mathrm{~cm}$, $\rho_{0}=2 \mathrm{~cm}$; this time it refers, however, to the TE case (Neumann boundary conditions): See the text. Again, the dotted line corresponds to $N=10$, and the solid line to $N=40$.

Fig.7 - In this figure, which refers to the TE case, two elements are depicted of the train 
of X-shaped pulses represented by eq.(16), with $\theta=60^{\circ}$ and $N=40$, while keeping the same $a$ and $b$ values as before.

Fig.8 - The orthogonal projection is shown of a single pulse (of the solution in Fig.7, for the TE case) onto the plane $(\rho, z)$ for $t=0$, with $\theta=60^{\circ}$ and $N=40$.

Fig.9 - Dispersion curves for the symmetrical $\mathrm{TM}_{0 n}$ modes in a perfect coaxial waveguide, and location of the frequencies whose corresponding modes possess the same phasevelocity. [Actually, the phase-velocity $c / \cos \theta$ of all the terms in expansion (12) is always the same, being independent of the mode index $n$ : In such a case, it is known that the group-velocity of the pulse (namely, the velocity tout court of the localized pulse) becomes equal to the phase-velocity.]

Figs.10 - Time evolution of a finite total energy solution. Choosing $q=0.606 \mathrm{~s}, c=1$, $N=40, a=1 \mathrm{~cm}, b=3 \mathrm{~cm}$ and $\theta=45^{\circ}$, there is only one X-shape pulse inside the subluminal envelope: see the text. The pulse and envelope velocities are given by $V=1 / \cos \theta$ and $v_{g}=1 / V$ : The superluminal speed $V=1 / v_{\mathrm{g}}$ of such a pulse can be regarded, of course, as the actual velocity of the wave. Figures (a), (b), (c), (d), (e) and (f) show a complete cycle of the pulse; they correspond to the time instants $t=0$, $t=0.5 \mathrm{~s}, t=1 \mathrm{~s}, t=3 \mathrm{~s}, t=3.5 \mathrm{~s}$, and $t=4 \mathrm{~s}$, respectively. 


\section{REFERENCES}

[1] M.Zamboni Rached, E.Recami and F.Fontana: "Localized Superluminal solutions to Maxwell equations propagating along a normal-sized waveguide" [Lanl Archives \# physics/0001039, Phys. Rev. E64 (2001) 066603.

[2] H.Bateman: Electrical and Optical Wave Motion (Cambridge Univ.Press; Cambridge, 1915), p.315. See also: J.A.Stratton: Electromagnetic Theory (McGraw-Hill; New York, 1941), p.356.

[3] A.O.Barut et al.: Phys. Lett. A143 (1990) 349; Found. Phys. Lett. 3 (1990) 303; Found. Phys. 22 (1992) 1267.

[4] A.O.Barut et al.: Phys. Lett. A180 (1993) 5; A189 (1994) 277.

[5] R.Donnelly and R.W.Ziolkowski: Proc. Roy. Soc. London A440 (1993) 541; I.M.Besieris, A.M.Shaarawi and R.W.Ziolkowski: J. Math. Phys. 30 (1989) 1254; S.Esposito: Phys. Lett. A225 (1997) 203; J.Vaz and W.A.Rodrigues: Adv. Appl. Cliff. Alg. S-7 (1997) 457.

[6] R.Courant and D.Hilbert: Methods of Mathematical Physics (J.Wiley; New York, 1966), vol.2, p.760. Cf. also: J.N.Brittingham: J. Appl. Phys. 54 (1983) 1179; R.W.Ziolkowski: J. Math. Phys. 26 (1985) 861; J.Durnin, J.J.Miceli and J.H.Eberly: Phys. Rev. Lett. 58 (1987) 1499; Opt. Lett. 13 (1988) 79; A.M.Shaarawi, I.M.Besieris and R.W.Ziolkowski: J. Math. Phys. 31 (1990) 2511; P.Hillion: Acta Applicandae Matematicae 30 (1993) 35.

[7] A.O.Barut, G. D.Maccarrone and E.Recami: Nuovo Cimento A71 (1982) 509; E.Recami et al.: Lett. Nuovo Cim. 28 (1980) 151; 29 (1980) 241.

[8] E.Recami: Rivista N. Cim. 9(6) (1986) 1-178. Cf. also E.Recami, F.Fontana and R.Garavaglia: Int. J. Mod. Phys. A15 (2000) 2793; E.Recami et al.: Il Nuovo Saggiatore 
$2(3)$ (1986) 20; 17(1-2) (2001) 21; and Found. Phys. 31 (2001) 1119.

[9] J.-y.Lu and J.F.Greenleaf: IEEE Trans. Ultrason. Ferroelectr. Freq. Control 39 (1992) 19.

[10] R.W.Ziolkowski, I.M.Besieris and A.M.Shaarawi: J. Opt. Soc. Am., A10 (1993) 75.

[11] E.Recami: Physica A252 (1998) 586. See also J.-y.Lu, J.F.Greenleaf and E.Recami: "Limited diffraction solutions to Maxwell (and Schroedinger) equations", Lanl Archives eprint physics/9610012 (Oct.1996). Cf. also E.Recami, in Time's Arrows, Quantum Measurement and Superluminal Behaviour, ed. by D.Mugnai, A.Ranfagni and L.S.Shulman (C.N.R.; Rome, 2001), pp.17-36.

[12] M.Zamboni Rached, E.Recami and H.E.Hernáqndez-Figueroa: "New localized Superluminal solutions to the wave equations with finite total energies and arbitrary frequencies", Lanl Archives e-print physics/0109062, to appear in Europ. Phys. Journal D.

[13] J.-y.Lu and J.F.Greenleaf: IEEE Trans. Ultrason. Ferroelectr. Freq. Control 39 (1992) 441 [in this case the beam speed is larger than the sound speed in the considered medium].

[14] P.Saari and K.Reivelt: Phys. Rev. Lett. 79 (1997) 4135.

[15] D.Mugnai, A.Ranfagni and R.Ruggeri: Phys. Rev. Lett. 84 (2000) 4830. [For a panoramic review of the "Superluminal" experiments, see E.Recami: Lanl Archives eprint physics/0101108, Found. Phys. 31 (2001) 1119].

[16] Cf. also, e.g., A.M.Shaarawi and I.M.Besieris, J. Phys. A: Math.Gen. 33 (2000) 7227; 33 (2000) 7255; 33 (2000) 8559; Phys. Rev. E62 (2000) 7415.

[17] See, e.g., R.Collins: Field Theory of Guided Waves (1991). 
[18] J.-y.Lu, H.-h.Zou and J.F.Greenleaf: IEEE Transactions on Ultrasonics, Ferroelectrics and Frequency Control 42 (1995) 850.

[19] See, e.g., E.Butkov: Mathematical Physics (Addison-Wesley; 1968).

[20] See, e.g., J.D.Jackson: Classical Electrodynamics (J.Wiley; New York, 1975).

[21] Cf., e.g., S.Ramo, J.R.Whinnery and T.Van Duzer: Fields and Waves in Communication Electronics, Chapt. 8 (John Wiley; New York, 1984).

[22] Cf., e.g., ref.[11] and refs. therein.

[23] See, e.g., M.Zamboni Rached and H.E.Hernández-Figueroa: Optics Comm. 191 (2000) 49. From the experimental point of view, cf., e.g., S.Longhi, P.Laporta, M.Belmonte and E.Recami: "Measurement of superluminal optical tunnelling in doublebarrier photonic bandgaps", Phys. Rev. E65 (2002) 046610. Cf. also V.S.Olkhovsky, E.Recami and G.Salesi: "Tunneling through two successive barriers and the Hartman (Superluminal) effect", Europhys. Lett. 57 (2002) 879-884; Y.Aharonov, N.Erez and B.Reznik: "Superoscillations and tunnelling times", Phys. Rev. A65 (2002) 052124.

[24] I.M.Besieris, M.Abdel-Rahman, A.Shaarawi and A.Chatzipetros: Progress in Electromagnetic Research (PIER) 19 (1998) 1-48 (1998). 
This figure "mkrhf1.jpg" is available in "jpg" format from: http://arxiv.org/ps/physics/0209104v1 
This figure "mkrhf2.jpg" is available in "jpg" format from: http://arxiv.org/ps/physics/0209104v1 
This figure "mkrhfo3a.jpg" is available in "jpg" format from: http://arxiv.org/ps/physics/0209104v1 
This figure "mkrhfo3b.jpg" is available in "jpg" format from: http://arxiv.org/ps/physics/0209104v1 
This figure "mkrhfo4.jpg" is available in "jpg" format from: http://arxiv.org/ps/physics/0209104v1 
This figure "mkrhfo5.jpg" is available in "jpg" format from: http://arxiv.org/ps/physics/0209104v1 
This figure "mkrhf6.jpg" is available in "jpg" format from: http://arxiv.org/ps/physics/0209104v1 
This figure "mkrhf7.jpg" is available in "jpg" format from: http://arxiv.org/ps/physics/0209104v1 
This figure "mkrhf8.jpg" is available in "jpg" format from: http://arxiv.org/ps/physics/0209104v1 
This figure "mkrhf9.jpg" is available in "jpg" format from: http://arxiv.org/ps/physics/0209104v1 
This figure "mkrhf10a.jpg" is available in "jpg" format from: http://arxiv.org/ps/physics/0209104v1 
This figure "mkrhf10b.jpg" is available in "jpg" format from: http://arxiv.org/ps/physics/0209104v1 
This figure "mkrhf10c.jpg" is available in "jpg" format from: http://arxiv.org/ps/physics/0209104v1 
This figure "mkrhf10d.jpg" is available in "jpg" format from: http://arxiv.org/ps/physics/0209104v1 
This figure "mkrhf10e.jpg" is available in "jpg" format from: http://arxiv.org/ps/physics/0209104v1 
This figure "mkrhf10f.jpg" is available in "jpg" format from: http://arxiv.org/ps/physics/0209104v1 